\title{
Cambios en la ingesta de sal y práctica de ejercicio físico en pacientes hipertensos por educación sanitaria en el primer nivel de atención de salud
}

Changes in salt ingestion and physical exercise in hypertensive patients by health education at the first level of health care attention

\author{
Arturo Gabriel Jurado-Vega ${ }^{1}$
}

\section{RESUMEN}

Aunque se asume que la educación sanitaria mejora los estilos de vida de los pacientes hipertensos, no existe suficiente bibliografía que apoye esta asociación. Objetivo: Evaluar el efecto de la educación sanitaria ofrecida a los pacientes hipertensos sobre sus prácticas de ingesta de sal y ejercicio físico. Material y métodos: Estudio descriptivo, transversal y basado en encuestas aplicadas a 179 pacientes hipertensos que acudieron entre junio y julio de 2009 a los servicios de medicina general, medicina interna y cardiología independientemente del motivo de atención. Se analizó con el programa SPSS versión 17.0 y aplicando el test de chi cuadrado. Resultados: El $67 \%$ fue del sexo femenino, $60,9 \%$ era mayor de 60 años y el 72,1\% tenía educación secundaria o superior. El $25 \%$ había recibido charlas y $22 \%$ material educativo en salud en los últimos 6 meses. No se encontró asociación significativa entre haber recibido educación sanitaria con la práctica de actividad física regular ni de menor ingesta de sal. Conclusiones: En este estudio, la educación sanitaria no tuvo influencia sobre la práctica de ejercicio físico ni sobre la ingesta de sal.

PALABRAS CLAVE: Educación sanitaria, hipertensión, ejercicio, cloruro de sodio. (Fuente: DeCS BIREME)

\section{SUMMARY}

Although it is assumed that health care education improves life style in hypertensive patients, there is no enough evidence to support its implementation. Objective: Evaluate the effect of health care education on salt ingestion and physical exercise in hypertensive patients. Methods: Descriptive, cross-sectional study based on surveys applied to 179 hypertensive patients who attended the internal medicine and cardiology outpatient clinics from June to July 2009. Chi square test was performed using the SPSS statistical package version 17.0. Results: $67 \%$ were females; $69 \%$ were older than 60 years of age; $72.1 \%$ had secondary school level or superior education level. Health care education was received by $25 \%$ of the participants, $22 \%$ had received printed material in the last 6 months. Having received health care education was not associated with less salt ingestion or with more physical exercise.

Conclusions: Health care education did not influence salt ingestion and physical exercise in this study.

KEY WORDS: Health education, hypertension, physical exercise, low-salt dietary. (Source: MeSH NLM) 


\section{INTRODUCCIÓN}

La adopción de estilos de vida saludables, como la reducción de la ingesta de sal y el ejercicio físico regular, es indispensable como parte del manejo de los sujetos hipertensos (1). Estas prácticas tienen los siguientes beneficios: la disminución de sal en la dieta reduce en $5 \mathrm{~mm} \mathrm{Hg}$ la presión arterial sistólica (PAS), la actividad física regular disminuye la PAS en $5 \mathrm{~mm} \mathrm{Hg}$ y la baja de peso la reduce en $3 \mathrm{~mm} \mathrm{Hg}$. Sin embargo, no basta con hacer ejercicio, se requiere que sea aeróbico, de aproximadamente 40 minutos por sesión y que se efectúe la mayoría de días de la semana. $(2,3)$ Si bien algunas personas tienen el hábito saludable de consumir una dieta baja en sal o hacer actividad física, esta práctica no es prevalente. Así, según un estudio descriptivo efectuado el 2003, encontramos que la frecuencia de actividad física regular en los mayores de 15 años en Lima metropolitana es de 10,6\% (4).

Se ha propuesto que la educación sanitaria puede contribuir a la adopción de estilos de vida saludables (5). Sin embargo, el brindar educación sin alguna otra intervención ha tenido poco éxito para mejorar la adherencia y control de la hipertensión (6). Igualmente, la distribución pasiva de material educativo impreso o audiovisual ha sido inefectiva en modificar la práctica profesional o en mejorar los resultados en salud (7).

En nuestro medio y particularmente en el ámbito de la atención primaria, existen escasos estudios sobre las características e impacto de la educación sanitaria brindada. El objetivo del estudio fue determinar si la educación sanitaria ofrecida a pacientes hipertensos atendidos en un establecimiento del primer nivel de atención tuvo efectos sobre sus prácticas de ingesta de sal y ejercicio físico.

\section{MATERIAL Y MÉTODOS}

Investigación operativa, descriptivo, transversal realizado en el Policlínico "Juan José Rodríguez Lazo", un establecimiento de atención primaria de EsSALUD, el cual sirve a los distritos de Chorrillos y Barranco. Dichos distritos están en el litoral limeño, en la parte sur de la ciudad, su población es urbana y en su mayor parte de clase media. Se incluyeron personas con edad entre 40 a 64 años con diagnóstico auto-referido de hipertensión arterial (HTA) y cuyo diagnóstico había sido realizado por un profesional de salud. No se tomó en cuenta el tiempo de diagnóstico.

Se excluyó a quienes presentaban deambulación asistida o no podían deambular en los últimos 6 meses y a los que no completasen la entrevista.

Muestra. Se tomó una muestra no probabilística y por conveniencia constituida por 179 pacientes que acudieron entre junio y julio de 2009 a los servicios de medicina general, medicina interna y cardiología independientemente del motivo de atención. No se consideró a pacientes que acudieron al servicio de emergencia.

\section{Definiciones operacionales}

Estilos de vida adoptados:

- Consumo de sal: se consideró "menor consumo de sal" cuando referían consumo bajo de sal 4 ó más veces por semana en los últimos 6 meses. El resto de casos se agrupó como nunca o irregularmente.

- Práctica de actividad física: se consideró "práctica de actividad física regular" cuando referían efectuar alguna actividad física 2 a más veces por semana en los últimos 6 meses. El resto de casos se agrupó como nunca o irregularmente.

\section{Educación sanitaria ofrecida:}

- Charlas educativas en salud recibidas. Se consideró que había recibido charla educativa cuando mencionaba haber recibido por lo menos una charla relacionada con HTA en los últimos 6 meses por cualquier miembro del equipo de salud.

- Material educativo en salud recibido. Se consideró que había recibido material educativo cuando mencionaba haber recibido trípticos, afiches, folletos, manuales o volantes relacionados a la hipertensión arterial en los últimos 6 meses procedente de alguna institución de salud.

Se aplicó un cuestionario no validado a cada paciente, el cual abarcó una diversidad de áreas temáticas como: características de los usuarios y la hipertensión, prácticas de estilos de vida y educación sanitaria sobre prácticas de estilos de vida.

\section{Análisis estadístico}

Los formatos de las encuestas de salida, fueron sometidos a procedimientos de limpieza de la información, luego se generó una base de datos con información válida que fue procesada empleando el programa estadístico SPSS versión 17. En un primer momento se calcularon las proporciones de los indicadores propuestos. Posteriormente se empleó el test de chi cuadrado para explorar la relación entre la 
educación sanitaria recibida, y la ingesta de sal y el ejercicio físico regular.

Un comité de ética evaluó el trabajo para garantizar la confidencialidad de la información y la seguridad de los participantes del estudio. Los participantes firmaron consentimiento informado antes de ser entrevistados.

Tabla 1. Características demográficas, socioeconómicas y de morbilidad en la población de estudio.

\begin{tabular}{lrc}
\hline \multicolumn{1}{l}{$\mathbf{n}$} & $\mathbf{\%}$ \\
\hline Sexo & 120 & 67,0 \\
Femenino & 59 & 33,0 \\
$\quad$ Masculino & & \\
Edad & 70 & 39,1 \\
40 a 60 años & 109 & 60,9 \\
$>60$ años & &
\end{tabular}

\section{Nivel educativo}

$\begin{array}{lcr}\text { Sin estudios } & 4 & 2,2 \\ \text { Primaria } & 46 & 25,7 \\ \text { Secundaria } & 66 & 36,9 \\ \text { Superior } & 63 & 35,2 \\ \text { Ocupación } & & \\ \text { Empleado(a) } & 34 & 19,0 \\ \text { Obrero(a) } & 30 & 16,8 \\ \text { Su casa } & 73 & 40,7 \\ \text { Jubilado(a) } & 42 & 23,5\end{array}$

Tiempo de diagnóstico de HTA (*)

$\begin{array}{lcc}<1 \text { año } & 22 & 12,3 \\ 1 \text { a } 5 \text { años } & 79 & 44,1 \\ 5 \text { a } 10 \text { años } & 32 & 17,9 \\ >10 \text { años } & 46 & 25,7 \\ \begin{array}{l}\text { Recibe medicación antihipertensiva } \\ \begin{array}{l}\text { Diagnóstico de sobrepeso u } \\ \text { obesidad (¥) }\end{array}\end{array} & 167 & 93,3 \\ \begin{array}{l}\text { Enfermedad que dificulte caminar } \\ (\boldsymbol{\alpha})\end{array} & 143 & 80,8 \\ \end{array}$

*,¥,\$,€: Se consideró dichos diagnósticos según referían que se los había informado un profesional de salud.

$€, ¥, \$$ : En estos casos las respectivas sumas no coinciden con el total de entrevistados debido a que algunos desconocían si tenían o no dichos problemas de salud.

$\alpha$ : Se incluyó enfermedades osteomusculares, cardiovasculares y pulmonares.

\section{RESULTADOS}

En la tabla 1 podemos ver las características demográficas, socioeconómicas y de morbilidad de la población entrevistada. La población fue predominantemente de mujeres y de mayores de 60 años (60\%). El 56\% tenía 5 años o menos de diagnóstico de HTA.

En la tabla 2 se muestran las características de las charlas en salud recibidas por la población de estudio; sólo $25 \%$ de entrevistados recibieron charlas en salud. De éstas algo más de la mitad estaban relacionadas a la HTA. Las sesiones educativas fueron realizadas en su mayoría por profesionales de enfermería y de la red de EsSALUD.

La tabla 3 muestra las características del material educativo que recibieron los pacientes, el 22\% recibió material educativo impreso, siendo el más frecuente los folletos y de la red EsSALUD.

Tabla 2. Características de las charlas en salud recibidas por la población de estudio $(\mathrm{n}=179)$.

\begin{tabular}{lll}
\hline Educación sanitaria en forma de charlas & n & $\%$ \\
\hline Recibieron charlas & 45 & 25
\end{tabular}

Temas de las charlas recibidas(*)

Sobre dieta para pacientes hipertensos

$\begin{array}{lr}21 & 40 \\ 8 & 15 \\ 3 & 6 \\ 9 & 17 \\ 3 & 6 \\ 9 & 17\end{array}$

Profesional que brindó la charla(€)

$\begin{array}{lcc}\text { Enfermera(o) } & 31 & 66 \\ \text { Médico(a) } & 6 & 13 \\ \text { Nutricionista } & 2 & 4 \\ \text { Otros } & 8 & 17\end{array}$

Afiliación del profesional que brindó la

charla

\begin{tabular}{lcc} 
EsSalud & 34 & 74 \\
MINSA & 4 & 9 \\
Otros & 8 & 17 \\
\hline
\end{tabular}

(*) Hubo encuestados que recibieron más de una charla.

$(€)$ Dos pacientes recibieron más de una charla, brindadas estas a su vez por distintos profesionales. 
Tabla 3. Características del material educativo en salud recibido por la población del estudio ( $\mathrm{n}=179)$.

\begin{tabular}{lcc}
\hline Educación sanitaria mediante material educativo & $\mathbf{n}$ & $\mathbf{\%}$ \\
\hline Total de personas que recibieron material educativo en los últimos $6 \mathrm{~m}$ & 40 & 22 \\
Tipo de material impreso educativo recibido & & \\
$\quad$ Tríptico & 6 & 15,0 \\
Folleto & 31 & 77,5 \\
Otros & 3 & 7,5
\end{tabular}

Institución de procedencia del material educativo recibido(*)

\begin{tabular}{lrr} 
EsSalud & 28 & 68,3 \\
MINSA & 4 & 9,8 \\
Otros & 9 & 21,9 \\
\hline
\end{tabular}

(*) Uno de los entrevistados recibió folletería educativa sobre la hipertensión arterial de EsSalud y de una clínica particular.

Tabla 4. Práctica de actividad física y consumo de sal en últimos 6 meses según educación sanitaria recibida por la población del estudio.

\begin{tabular}{|c|c|c|c|c|c|c|c|c|c|c|c|c|}
\hline & \multicolumn{4}{|c|}{ Charla educativa en salud } & \multirow{2}{*}{ OR } & \multirow{2}{*}{ IC } & \multicolumn{4}{|c|}{ Material educativo en salud } & \multirow{2}{*}{ OR } & \multirow{2}{*}{ IC } \\
\hline & Sí & $\%$ & No & $\%$ & & & Sí & $\%$ & No & $\%$ & & \\
\hline \multicolumn{13}{|l|}{$\begin{array}{l}\text { Práctica de actividad física } \\
\text { regular en últimos } 6 \text { meses }\end{array}$} \\
\hline $\begin{array}{l}\text { La mayor parte de las } \\
\text { semanas }\end{array}$ & 17 & 25 & 51 & 75 & \multirow{2}{*}{$0,95^{*}$} & \multirow{2}{*}{$0,47-1,92$} & 20 & 29 & 48 & 71 & \multirow{2}{*}{$1,99 *$} & \multirow{2}{*}{$0,96-4,12$} \\
\hline Nunca o irregularmente & 27 & 26 & 77 & 74 & & & 18 & 17 & 86 & 83 & & \\
\hline \multicolumn{13}{|l|}{$\begin{array}{l}\text { Práctica de menor consumo } \\
\text { de sal en últimos } 6 \text { meses }\end{array}$} \\
\hline $\begin{array}{l}\text { La mayor parte de las } \\
\text { semanas }\end{array}$ & 31 & 25 & 92 & 75 & \multirow{2}{*}{$1,01^{*}$} & \multirow{2}{*}{$0,49-2,12$} & 28 & 23 & 95 & 77 & \multirow{2}{*}{$1,08^{*}$} & \multirow{2}{*}{$0,50-2,32$} \\
\hline Nunca o irregularmente & 14 & 25 & 42 & 75 & & & 12 & 21 & 44 & 79 & & \\
\hline
\end{tabular}

*p= no significativo

Tabla 5. Práctica de actividad física y consumo de sal en últimos 6 meses entre los que recibieron charlas y material educativo simultáneamente

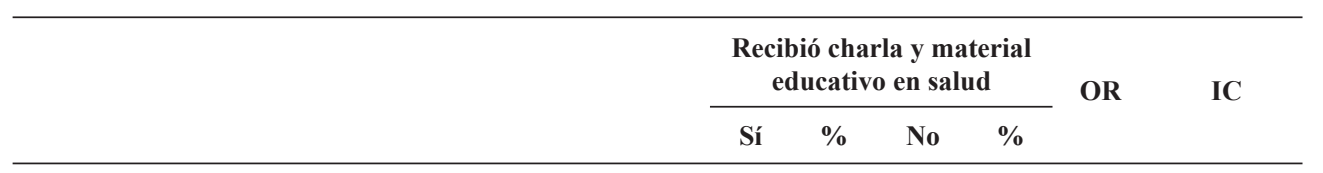

Práctica de actividad física regular en últimos 6 meses

La mayor parte de las semanas

Nunca o irregularmente

Práctica de menor consumo de sal en últimos 6 meses

\begin{tabular}{|c|c|c|c|c|c|c|}
\hline La mayor parte de las semanas & 19 & 19 & 83 & 81 & $1,6^{*}$ & $0,55-4,63$ \\
\hline Nunca o irregularmente & 5 & 13 & 35 & 87 & & \\
\hline
\end{tabular}

$\begin{array}{llllll}11 & 21 & 42 & 79 & & \\ 12 & 14 & 71 & 86 & & \end{array}$

$* \mathrm{p}=$ no significativo 
Al comparar si la práctica de actividad física regular estaba asociado a la educación sanitaria recibida a través de charlas y material educativo en salud, no se encontró asociación estadística entre la práctica de actividad física regular con haber recibido charlas educativas $(\mathrm{OR}=0,95 ;[0,47-1,92])$ ni con haber recibido material educativo $(\mathrm{OR}=1,99 ;[0,96-$ $4,12])$. Tampoco se encontró asociación significativa entre la práctica de menor consumo de sal con haber recibido charlas educativas $(\mathrm{OR}=1,01 ;[0,49-2,12])$ o haber recibido material educativo $(\mathrm{OR}=1,08 ;[0,50$ 2,32]) (Tabla 4).

No se encontró asociación entre la práctica de un menor consumo de sal en los últimos 6 meses y haber recibido charlas y material educativo simultáneamente $(\mathrm{OR}=1,6 ;[0,55-4,63])$, asimismo, no se encontró asociación entre la práctica de actividad física regular en los últimos 6 meses y haber recibido charlas y material educativo simultáneamente $(\mathrm{OR}=1,54 ;[0,63$ $3,82]$ ) (Tabla 5).

Por otro lado, tampoco se encontró asociación entre ser obeso o tener sobrepeso con la práctica de actividad física regular.

\section{DISCUSIÓN}

En el estudio se exploró la asociación entre la educación sanitaria - en forma de charlas y material educativo - y la adopción de estilos de vida saludables, en pacientes con HTA de un establecimiento de atención primaria. No se encontró diferencias significativas en la práctica de actividad física o de menor ingesta de sal entre los que recibieron educación sanitaria y quienes no la recibieron.

Nuestros resultados coinciden con los del estudio de Magadza et al (8), quien encontró que la combinación de charlas, encuentros mensuales y material educativo referido a la terapia incrementaron el conocimiento sobre la hipertensión y sus creencias sobre las medicinas $(p<0,0001)$ pero no repercutió sobre sus estilos de vida ni adherencia. Sin embargo, existen estudios en los que encontraron efectos positivos de la intervención educativa, en el programa de mejoramiento del tratamiento hipertensivo (PROMETA) encontraron que la PAS disminuyó significativamente (9) y en Hypertension Detection and folow-up Program (HDFP), una disminución de la mortalidad por todas las causas $(7,10,11)$.
En EsSALUD las actividades educativas que intentan cambios de conducta del paciente están a cargo de un equipo multidisciplinario. Estas actividades incluyen la prescripción de actividad física, charlas, talleres, entrega de material educativo y orientación y soporte nutricional (12). Sin embargo, la calidad con la que se ofrecen estas acciones es heterogénea, y no se percibe articulación entre las diferentes actividades educativas, todo lo cual podría estar influyendo en los resultados alcanzados.

También se debe tener en cuenta que el $60 \%$ de los entrevistados era mayor de 60 años, lo que podría estar influyendo en una menor actividad física $(13,14)$.

Aunque de los resultados se podría asumir un escaso impacto de las actividades educativas en la adopción de estilos de vida saludables, debemos considerar algunas limitaciones del diseño que podrían estar influyendo en los resultados. En primer lugar, el diseño de estudio transversal, no permite medir el impacto de la educación sanitaria impartida, para esto se emplean preferentemente estudios longitudinales (15). Además, la encuesta al no ser anónima, puede inducir a los entrevistados a emitir respuestas que consideran es de interés del investigador. Otra limitación del estudio fue haber utilizado un instrumento no validado completamente.

En conclusión, este estudio muestra que en condiciones habituales en establecimientos de atención primaria, la educación sanitaria para pacientes hipertensos es insuficiente, y tiene poco efecto en la adopción de prácticas saludables de vida. Las explicaciones pueden ser múltiples, pero este estudio no ha incidido en una evaluación de esa naturaleza. Dado que existe una posibilidad razonable que los pobres resultados se deban a la cantidad o calidad de las acciones de educación sanitaria en los establecimientos de atención primaria, es recomendable fortalecer estas acciones buscando mejores resultados en el futuro. Finalmente, debe resaltarse que las actividades educativas más recomendadas para generar el cambio de actitud y comportamiento son el diálogo y trabajo en grupo (5) y no la oferta pasiva de charlas y material educativo.

\section{Correspondencia:}

Arturo Jurado Vega

Jirón San Miguel 242, Urb. J.C. Mariátegui, Villa María del Triunfo. Lima, Perú.

Correo electrónico: arturomh98sp@yahoo.es 


\section{REFERENCIAS BIBLIOGRÁFICAS}

1. Colleen K, Blackwell J. What lifestyle changes should we recommend for the patient with newly diagnosed hypertension? The Journal of Family Practice. 2006; 55(11):991-3.

2. Lochner J, Rugge B, Judkins D. How effective are lifestyle changes for controlling hypertension? The Journal of Family Practice. 2005;5(1):73-4.

3. National High Blood Pressure Education Program. The Seventh Report of teh Joint National Committee on Prevention, Detection, Evaluation and Treatment on Hight Blood Pressure. Washington D.C.: National Institutes of Health; 2004.

4. Seclén-Palacín J, Jacoby E. Factores demográficos y ambientales asociados con la actividad física deportiva en la población urbana del Perú. Rev Panam Salud Pública. 2003;14(4):255-64.

5. Trilla-Soler MC, Plana-Fernández $\mathrm{M}$, Espluga Capdevila A. Educación Sanitaria. Atención Primaria: conceptos, organización y práctica clínica.Barcelona: Elsevier España, S.L; 2008.

6. Schroeder K, Fahey T, Ebrahim S. Intervenciones para mejorar el cumplimiento del tratamiento en pacientes con hipertensión arterial en ámbitos ambulatorios (Revisión Cochrane traducida). En: La Biblioteca Cochrane Plus, 2008 Número 4. Oxford: Update Software Ltd. Disponible en: http://www.updatesoftware.com.

7. Althabe F, Bergel E, Cafferata ML, et al. Strategies for improving the quality of health care in maternal and child health in low- and middle-income countries: an overview of systematic reviews. Paediatr Perinat Epidemiol. 2008; 22(S1): 42-60.

8. Magadza C, Radloff SE, Srinivas SC. The effect of an educational intervention on patients' knowledge about hypertension, beliefs about medicines, and adherence. Res Social Adm Pharm. 2009;5(4):36375.

9. Mariño-Arroyo R. Determinación del efecto del programa de mejoramiento del tratamiento antihipertensivo (prometa) sobre los conocimientos básicos de la hipertensión, índice de masa corporal y control de la presión arterial en pacientes hipertensos expuestos a este programa. Tesis de Bachiller en Medicina. Lima, Perú: Universidad Peruana Cayetano Heredia, 2003. 37 pp.

10. Fahey T, Ebrahim S, Schroeder K. Educational and organizational interventions used to improve the management of hypertension in primary care: a systematic review. British Journal of General Practice. 2005;55:875-82.

11. Salleras Sanmartí L. Educación Sanitaria: Principios, métodos y aplicaciones. Barcelona: Ediciones Díaz de Santos; 1985.

12. Gerencia Central de Prestaciones de Salud, EsSALUD. Cartera de Servicios de Atención Primaria de EsSalud. Lima, Perú: EsSalud; 2008.

13. Fernández-Larrea N, Clúa-Calderín A, Baez-Dueñas R, Ramírez-Rodríguez M, Prieto-Díaz B. Estilos de Vida, Bienestar Subjetivo y Salud de los Ancianos. Rev Cubana Med Gen Integr. 2000;16(1):6-12.

14. Nied R, Franklin B. Promoting and Prescribing Exercise for the elderly. American Family Physician. 2002;65(3):419-26.

15. Fahey T, Ebrahim S, Schroeder K. Intervenciones para mejorar el control de la presión arterial en pacientes hipertensos. Revisión Cochrane traducida. La Biblioteca Cochrane Plus, 2007. Report No: 4.

Recibido: 16/06/12

Aceptado: 07/02/2013 